\title{
Sjögren's Syndrome Associated With Thrombotic Thrombocytopenic Purpura: A Case-Based Review
}

Jozélio F. Carvalho (1] · Yehuda Shoenfeld

Received: November 2, 2020 / Accepted: November 26, 2020 / Published online: December 14, 2020

(C) The Author(s) 2020

\begin{abstract}
Objective: To review all published cases of the rare association between thrombotic thrombocytopenic purpura (TTP) and Sjögren's syndrome (SS). The authors report an additional case of this unique association.

Methods: Systematic review of the literature and a case report. The database were articles published in PubMed/MEDLINE, Web of Science, LILACS, and SciELO, registered from 1966 to August 2020. The DESH terms were "Sjögren's syndrome" and "thrombotic thrombocytopenic purpura," without language limitation.
\end{abstract}

J. F. Carvalho $(\bowtie)$

Institute for Health Sciences from Federal University of Bahia, Salvador, Bahia, Brazil

e-mail: jotafc@gmail.com

Y. Shoenfeld

Chaim Sheba Medical Center The Zabludowicz

Center for Autoimmune Diseases, Tel Hashomer, Israel

Y. Shoenfeld

I.M. Sechenov First Moscow State Medical

University of the Ministry of Health of the Russian

Federation (Sechenov University), Saint Petersburg,

Russia
Results: Most patients were female (88\%), and the age varied from 30 to 75 years old. Concerning the sequence of disease appearance, SS followed by TTP was seen in seven articles, TTP and SS in three, and simultaneous appearance of both diseases in three studies. Primary SS was observed in 16 patients, and secondary SS was detected in two cases: dermatomyositis and rheumatoid arthritis. Anemia was the most common TTP manifestation, followed by thrombocytopenia, fever, consciousness alteration, renal impairment, and schistocytes' appearance on a blood smear. Treatment involved plasmapheresis, plasma exchange, rituximab, glucocorticoid, and cyclophosphamide. A good outcome was noted in most studies; few patients died.

Conclusions: TTP is a rare manifestation associated with SS. After the TTP diagnosis, plasmapheresis and/or plasma exchange should be immediately implemented.

Keywords: Autoantibodies; Autoimmunity; Sicca syndrome; Thrombosis; Thrombotic thrombocytopenic purpura; Sjögren's syndrome 


\section{Key Summary Points}

Sjögren's syndrome (SS) associated with thrombotic thrombocytopenia purpura (TTP) is a rarity.

We performed a systematic review on all published cases of this rare association.

Most cases are female and with primary SS, and also SS appeared commonly first than TTP.

Most cases were successfully treated with glucocorticoid, plasma exchange, and plasmapheresis. disintegrin and metalloproteinase with a thrombospondin type 1 motif, member 13), caused by anti-ADAMTS13 antibodies, leading to the formation of platelet-rich thrombi in the microvasculature. Clinically, it is characterized by hemolytic anemia and thrombocytopenia owing to microangiopathy anemia, consciousness alterations, fever, and renal impairment [3]. Some reports of TTP with SS are available in the literature with these conditions [4-16].

This article aims to report an additional case of a patient with TTP, subsequently diagnosed as primary SS. Furthermore, an extensive review of the literature on SS and TTP was performed.

\section{METHODS}

\section{Literature Review}

A systematic research of articles published in PubMed/MEDLINE, Web of Science, LILACS, and SciELO, registered from 1966 to August 2020, was performed. All the researched articles are based on the following DESH terms "Sjögren's syndrome" and "thrombotic thrombocytopenic purpura" without language limitation. Furthermore, a detailed case report on a patient with TTP subsequently diagnosed with SS is herein reported. The following parameters were screened for the published cases of SS and TTP association: demographic characteristics (gender, age), clinical features (clinical presentation of TTP, SS, and SS-related antibodies detection, the onset of symptoms and progression), therapy provided, and the response to this therapy. This article followed the PRISMA guidelines [17]. Informed consent was obtained from the patient.

\section{Case Report}

A 30-year-old Caucasian and black mixed ethnicity female patient with a past medical history of convulsion since in her childhood has been treated until now with carbamazepine. She was admitted in April of 2014 with petechiae over her legs and dark urine, and thrombocytopenia (platelets $5000 / \mathrm{mm}^{3}$ ). The patient received 
methylprednisolone pulse therapy $1 \mathrm{~g}$ daily for 3 days, and the platelets increased to 20,000/ $\mathrm{mm}^{3}$. During the hospital stay, she had three absence episodes and severe hypotension and was transferred to the intensive care unit (ICU). In that place, severe hemolytic anemia (hemoglobin $5 \mathrm{~g} / \mathrm{dl}$ ), thrombocytopenia, renal impairment, and oscillations of conscience level were verified with schizocytes. A diagnosis of thrombotic thrombocytopenic purpura was determined, and plasmapheresis was started. She received 15 sessions of plasmapheresis, and 1 month later, rituximab was infused. After this approach, the TTP improved, and the patient recovered from this condition. Due to antinuclear antibody positivity with a titer of $1: 640$, a diagnosis of lupus was considered, and hydroxychloroquine $400 \mathrm{mg} /$ day was prescribed. However, she did not have any evidence of skin lesions, serositis, glomerulonephritis, or polyarthritis. She has a cousin with lupus and an aunt with rheumatoid arthritis. After discharge, she went to our private clinic, and her physical examination was unremarkable. No sign of carbamazepine intoxication was found, such as gingival hypertrophy. Laboratory tests demonstrated positive antinuclear antibodies with a titer of 1:640, and a speckled pattern on indirect immunofluorescence in HEp2 cells. Anti-Ro/SSA, anti-La/SS-B, anti-RNP, anti-Sm, anti-dsDNA, lupus anticoagulant, IgG, IgM, and IgA anticardiolipin, anti-thyroperoxidase, ANCA, antithyroglobulin; IgA and IgG anti-gliadin, antiendomysium and anti-tissue transglutaminase antibodies and cryoglobulins were not detected. Complement levels were within normal range (CH50 $97 \mathrm{U} / \mathrm{ml}, \mathrm{C} 3150 \mathrm{mg} / \mathrm{dl}$, and C4 $30 \mathrm{mg} /$ dl). Blood cell count, creatinine, and urine spot were normal. Tests for thrombophilia were all negative or within normal range: factor V Leiden, mutant prothrombin, antithrombin III, proteins $\mathrm{C}$ and $\mathrm{S}$, and homocysteine. Serology for infectious diseases was negative: HIV 1 and 2, HTLV I and II, syphilis, cytomegalovirus, and hepatitis $B$ and $C$ viruses. Hemolysis test was negative at that moment, with a negative Coombs test, haptoglobin $240 \mathrm{mg} / \mathrm{dl}$ (25-190 mg/dl), normal bilirubin, and negative for schistocytes. ADAMTS 13 activity was less than $2 \%(>50 \%)$ and ADAMTS13 antigen was 0.1 (0.6-1.6). She complained of xerostomia and xerophthalmia. Salivary gland scintigraphy showed a decrease in salivary production bilaterally on submandibular and parotid glands. Schirmer test was $<5 \mathrm{~mm}$ in $5 \mathrm{~min}$ in both eyes, and Bengal rose tests were positive with a score $>5$. A minor salivary gland biopsy was positive with focal lymphocytic sialadenitis and a focus score of 1 foci $/ 4 \mathrm{~mm}$. A diagnosis of primary Sjögren's syndrome was then determined. Hydroxychloroquine was kept, and ocular lubricants were added. She is currently asymptomatic; under HCQ and lubricants, routine laboratory tests are within the normal range.

\section{RESULTS}

A total of 13 articles were found with 17 patients with SS and TTP. The analysis was performed with these 18 patients, including our case report.

Table 1 summarizes all cases of SS associated with TTP published in the literature, and our case report was added.

Regarding country of publication, the most cases came from Japan $(n=5)$, followed by China $(n=2)$, United States $(n=2)$, Norway $(n=1)$, Taiwan $(n=1)$, Israel $(n=1)$, Australia $(n=1)$, and Brazil $(n=1)$. Most patients were female $15 / 17$ (88\%), and age varied from 30 to 75 years old.

About the sequence of disease appearance, SS followed by TTP was seen in seven articles, TTP and then SS in three, and simultaneous appearance of both diseases in three studies.

Primary SS was observed in 16/18 (88.9\%) patients, and secondary SS was detected in $2 / 18$ (11.1\%) cases: dermatomyositis $(n=1)$ and rheumatoid arthritis $(n=1)$.

Anemia was seen in 16/18 (88.9\%) patients, thrombocytopenia in $15 / 18$ (83.3\%), fever in $6 / 18(33.3 \%)$, consciousness alteration in $8 / 18$ (44.4\%), renal impairment in 7/18 (38.9\%), and schistocytes in 7/18 (38.9\%).

SS-related autoantibodies were distributed as follows: ANA in 13 articles, anti-Ro/SS-A in 12, 


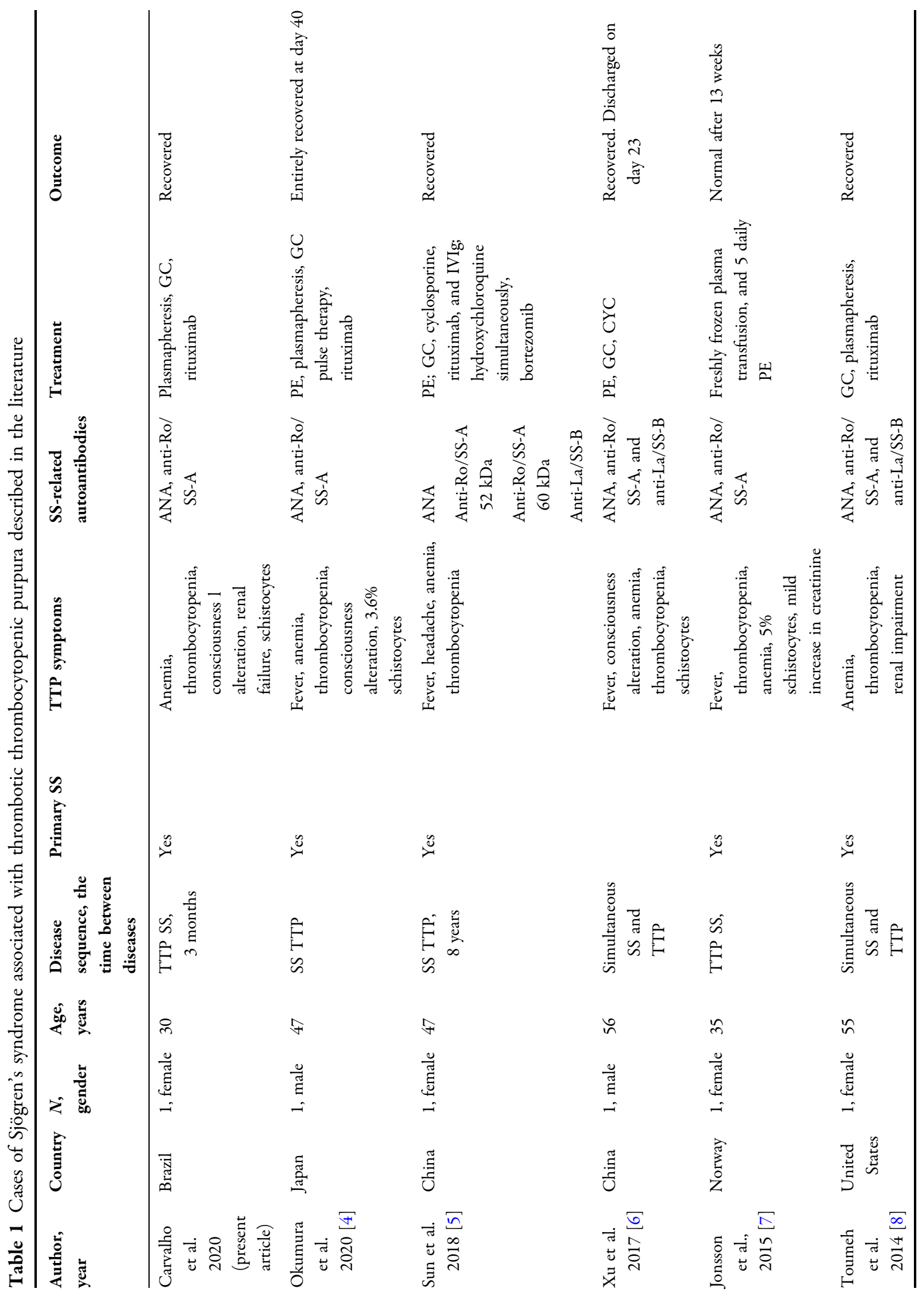




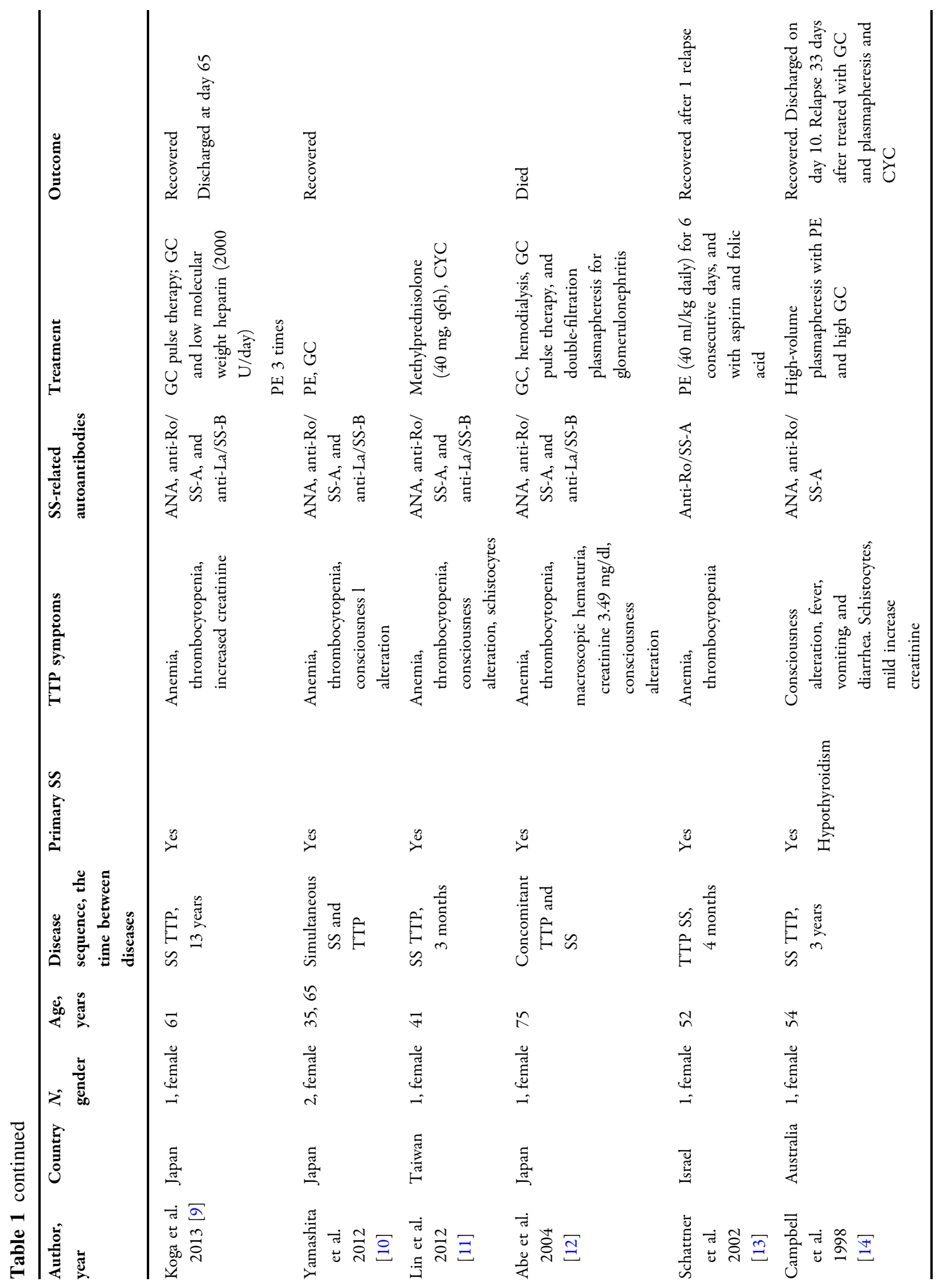




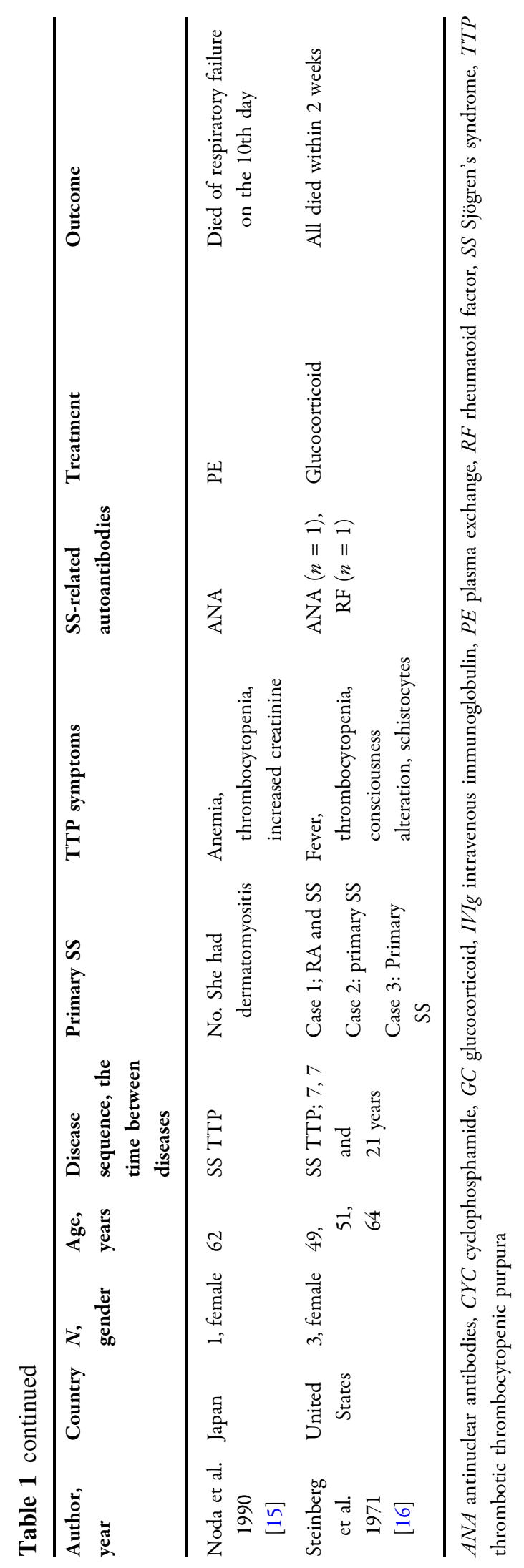


anti-La/SS-B in seven, and rheumatoid factor in one.

Treatment involved plasmapheresis in six studies, plasma exchange in eight articles, rituximab in four, glucocorticoid in 11, and cyclophosphamide in two.

A good outcome was noted in 11 articles, and patients died in three articles with four patients. In one study (1971), two patients died; neither plasmapheresis nor plasma exchange was used.

\section{DISCUSSION}

This article is an additional report of a patient who suffered from TTP and subsequently had a SS diagnosis. We followed the PRISMA guidelines [17], which is strongly recommended in a systematic review analysis.

Most patients had SS before the TTP development. This suggests that SS may have a pathophysiological role in initiating the microangiopathy. It is known that SS may present with vasculitis and the presence of several autoantibodies. SS has classical autoantibodies like antinuclear antibodies, anti-Ro/SS-A, and anti-La/Ss-b, a new article describes 19 novel autoantibodies in SS [18]. It will not be a surprise if investigated anti-ADAMTS13 antibodies might be found in SS without TTP.

The TTP initial diagnosis should be based on the history, physical examination, routine laboratory tests, and schistocytes definitive presence on the peripheral blood smear. Patients generally present with signs and symptoms and have laboratory abnormalities that reflect the underlying microvascular thrombi. A low baseline ADAMTS13 activity of $10 \%$ or less, as observed in our patient, with or without an anti-ADAMTS13 autoantibody, in the association with thrombocytopenia microangiopathic hemolytic anemia are highly suggestive of TTP. However, the classic "pentad" of clinical features is very typical for TTP, and it may appear in only $5 \%$ of patients. Therefore, after the TTP's clinical suspicion, rapid treatment is mandatory, even without ADAMTS13 levels detection. The treatment of TTP involves plasma exchange and glucocorticoids. When the disease is refractory to these therapeutical agents, the physician may increase plasma exchange twice daily, add rituximab, methylprednisolone pulse therapy, cyclosporine, and cyclophosphamide vincristine, and splenectomy [19].

TTP's risk factors are genetics that includes the black race, probably due to the lower frequency of the protective allele for TTP, HLADRB1*04. Interestingly, black people with TTP may better survive than white patients, despite comparable disease severity [20]. Our patient was Caucasian and black mixed ethnicity and it might explain her risk for TTP and good survival. In lupus patients, lymphopenia, high SLEDAI score 3 months before hospitalization, low hemoglobin levels, low levels of indirect bilirubin, and less severe thrombocytopenia were all independent risk factors in these patients for TTP development [21]. Moreover, in another study, the multivariate analysis showed that infection was the only independent risk factor for mortality in lupus patients with TTP [22]. In a different non-autoimmune condition, post-bone marrow transplant, patients who developed TTP were three times older and more twice as likely to be female compared to those cases who had not evolved to TTP [23]. Another study found that the black race and blood group $\mathrm{O}$ are independent risk factors for TTP [24]. Relying on these previous studies in other diseases, which evolved to TTP, female sex, old age, disease activity, blood group $\mathrm{O}$, and infections, seem to be the central risk factors for TTP. These findings agree with the present review since most SS patients associated with TTP were female, and 10/17 were more than 50 years old. However, the articles do not describe an infection or SS disease activity.

\section{CONCLUSIONS}

In conclusion, this systematic review analyzed the rarity of SS associated with TTP, and verified that most cases are female and with primary SS, and also SS appeared more commonly before TTP development. Most cases were successfully treated with glucocorticoids, plasma exchange, and plasmapheresis. 


\section{ACKNOWLEDGEMENTS}

The authors would like to thank Sergio Ribeiro for the English revision of the manuscript.

Funding. No funding or sponsorship was received for this study or publication of this article.

Authorship. All named authors meet the International Committee of Medical Journal Editors (ICMJE) criteria for authorship for this article, take responsibility for the integrity of the work as a whole, and have given their approval for this version to be published.

Disclosures. Jozélio F. Carvalho is on the editorial board for Rheumatology and Therapy but has nothing else to disclose. Yehuda Shoenfeld has nothing to disclose.

Compliance with Ethics Guidelines. The authors declare that the World Medical Association Declaration of Helsinki were followed. Informed consent was obtained from the patient for publication of this study.

Data Availability. Data sharing is not applicable to this article, as no datasets were generated or analyzed during the current study. We thank the patient, participant of this study.

Open Access. This article is licensed under a Creative Commons Attribution-NonCommercial 4.0 International License, which permits any non-commercial use, sharing, adaptation, distribution and reproduction in any medium or format, as long as you give appropriate credit to the original author(s) and the source, provide a link to the Creative Commons licence, and indicate if changes were made. The images or other third party material in this article are included in the article's Creative Commons licence, unless indicated otherwise in a credit line to the material. If material is not included in the article's Creative Commons licence and your intended use is not permitted by statutory regulation or exceeds the permitted use, you will need to obtain permission directly from the copyright holder. To view a copy of this licence, visit http://creativecommons.org/licenses/by$\mathrm{nc} / 4.0 /$.

\section{REFERENCES}

1. Both T, Dalm VA, van Hagen PM, van Daele PL. Reviewing primary Sjögren's syndrome: beyond the dryness-from pathophysiology to diagnosis and treatment. Int J Med Sci. 2017;14(3):191-200.

2. Fox RI. Sjögren's syndrome. Lancet. 2005;366(9482):321-31.

3. Chiasakul T, Cuker A. Clinical and laboratory diagnosis of TTP: an integrated approach. Hematol Am Soc Hematol Educ Program. 2018;2018(1): 530-8.

4. Okumura T, Hashimoto K, Aomura D, et al. Thrombotic thrombocytopenic purpura treated with rituximab associated with primary Sjögren's syndrome and primary hypothyroidism. Intern Med. 2020;59(5):715-9.

5. Sun R, Gu W, Ma Y, Wang J, Wu M. Relapsed/refractory acquired thrombotic thrombocytopenic purpura in a patient with Sjögren syndrome: Case report and review of the literature. Medicine (Baltimore). 2018;97(43):e12989.

6. Xu X, Zhu T, Wu D, Zhang L. Sjögren's syndrome initially presented as thrombotic thrombocytopenic purpura in a male patient: a case report and literature review. Clin Rheumatol. 2018;37(5): 1421-6.

7. Jonsson MK, Hammenfors D, Oppegaard O, Bruserud $\varnothing$, Kittang AO. A 35-year-old woman with influenza A-associated thrombotic thrombocytopenic purpura. Blood Coagul Fibrinolysis. 2015;26(4):469-72.

8. Toumeh A, Josh N, Narwal R, Assaly R. Refractory thrombotic thrombocytopenic purpura associated with primary Sjogren syndrome treated with rituximab: a case report. Am J Ther. 2014;21(2):e56-60.

9. Koga T, Yamasaki S, Nakamura H, et al. Renal thrombotic microangiopathies/thrombotic thrombocytopenic purpura in a patient with primary Sjögren's syndrome complicated with IgM monoclonal gammopathy of undetermined significance. Rheumatol Int. 2013;33(1):227-30.

10. Yamashita $H$, Takahashi $Y$, Kaneko $H$, Kano T, Mimori A. Thrombotic thrombocytopenic purpura with an autoantibody to ADAMTS13 complicating 
Sjögren's syndrome: two cases and a literature review. Mod Rheumatol. 2013;23(2):365-73.

11. Lin TY, Chang CC, Chang CC, Yuan JY, Chen HH. Cyclophosphamide-rescued plasmapheresis-unresponsive secondary thrombotic thrombocytopenic purpura caused by Sjögren's syndrome. Arch Med Sci. 2012;8(5):934-8.

12. Abe $\mathrm{H}$, Tsuboi $\mathrm{N}$, Yukawa $\mathrm{S}$, et al. Thrombotic thrombocytopenic purpura complicating Sjögren's syndrome with crescentic glomerulonephritis and membranous nephritis. Mod Rheumatol. 2004;14(2):174-8.

13. Schattner A, Friedman J, Klepfish A. Thrombotic thrombocytopenic purpura as an initial presentation of primary Sjögren's syndrome. Clin Rheumatol. 2002;21(1):57-9.

14. Campbell GN, Gallo JH. Relapsing thrombotic thrombocytopenic purpura (TTP) in Sjögren's syndrome. Aust N Z J Med. 1998;28(2):214.

15. Noda M, Kitagawa M, Tomoda F, Iida H. Thrombotic thrombocytopenic purpura as a complicating factor in a case of polymyositis and Sjögren's syndrome. Am J Clin Pathol. 1990;94(2):217-21.

16. Steinberg AD, Green WT Jr, Talal N. Thrombotic thrombocytopenic purpura complicating Sjögren's syndrome. JAMA. 1971;215(5):757-61.

17. Shamseer L, Moher D, Clarke M, et al. Preferred reporting items for systematic review and metaanalysis protocols (PRISMA-P) 2015: elaboration and explanation [published correction appears in BMJ. 2016 Jul 21;354:i4086]. BMJ. 2015;350:7647.
18. Martín-Nares E, Hernández-Molina G. Novel autoantibodies in Sjögren's syndrome: a comprehensive review. Autoimmun Rev. 2019;18(2):192-8.

19. Sayani FA, Abrams CS. How I treat refractory thrombotic thrombocytopenic purpura [published correction appears in Blood. 2017 Oct 5;130(14 ): 1684]. Blood. 2015;125(25):3860-7.

20. Martino S, Jamme M, Deligny C, et al. Thrombotic thrombocytopenic purpura in black people: impact of ethnicity on survival and genetic risk factors. PLOS ONE. 2016;11(7):e0156679.

21. Merayo-Chalico J, Demichelis-Gómez R, RajmeLópez S, et al. Risk factors and clinical profile of thrombotic thrombocytopenic purpura in systemic lupus erythematosus patients. Is this a distinct clinical entity in the thrombotic microangiopathy spectrum?: a case-control study. Thromb Res. 2014;134(5):1020-7.

22. Kwok SK, Ju JH, Cho CS, Kim HY, Park SH. Thrombotic thrombocytopenic purpura in systemic lupus erythematosus: risk factors and clinical outcome: a single centre study. Lupus. 2009;18(1): $16-21$.

23. Fuge R, Bird JM, Fraser A, et al. The clinical features, risk factors, and outcome of thrombotic thrombocytopenic purpura occurring after bone marrow transplantation. Br J Haematol. 2001;113(1):58-64.

24. Terrell DR, Motto DG, Kremer Hovinga JA, Lämmle $\mathrm{B}$, George JN, Vesely SK. Blood group O and black race are independent risk factors for thrombotic thrombocytopenic purpura associated with severe ADAMTS13 deficiency. Transfusion. 2011;51(10): 2237-43. 